Pure and Applied Mathematics Quarterly

Volume 7, Number 3

(Special Issue: In honor of

Jacques Tits)

$539-557,2011$

\title{
Can An Anisotropic Reductive Group Admit A Tits System?
}

\author{
Pierre-Emmanuel Caprace* and Timothée Marquis \\ Dedicated to Jacques Tits in honour of his 80th birthday
}

\begin{abstract}
Seeking for a converse to a well-known theorem by Borel-Tits, we address the question whether the group of rational points $G(k)$ of an anisotropic reductive $k$-group can admit a split spherical BN-pair. We show that if $k$ is a perfect field or a local field, then such a BN-pair must be virtually trivial. We also consider arbitrary compact groups and show that the only abstract BN-pairs they can admit are spherical, and even virtually trivial provided they are split.
\end{abstract}

Keywords: BN-pair, building, anisotropic reductive group, compact group

\section{INTRODUCTION}

In a seminal paper [5], Armand Borel and Jacques Tits established - amongst other things - that the group $G(k)$ of $k$-rational points of a (connected) reductive linear algebraic $k$-group $G$ always possesses a canonical BN-pair, where $k$ is an arbitrary ground field. More precisely, they showed that if $P$ is a minimal parabolic $k$-subgroup of $G$, and if $N$ is the normalizer in $G$ of some maximal $k$-split

Received August 19, 2009.

2000 Mathematics Subject Classfication. 20E42, 20G15 (Primary), 22C05 (Secondary).

*Supported by the Fund for Scientific Research-F.N.R.S., Belgium. 
torus contained in $P$, then $(P(k), N(k))$ is a BN-pair for $G(k)$. This result constitutes a cornerstone in understanding the abstract group structure of the group of $k$-rational points $G(k)$. As an application, it yields for example the celebrated simplicity result of Tits [19]. Of course, the aforementioned BN-pair is trivial when $G$ is anisotropic over $k$. (Abusing slightly the standard conventions, we shall say that $G$ is anisotropic if it has no proper $k$-parabolic subgroup, i.e. if $P=G$. As is well-known, this definition coincides with the standard one in case $G$ is semi-simple (see $[4,11.21])$ ). In fact, the abstract group structure of $G(k)$ remains intriguing and mysterious to a large extent in the anisotropic case. In this context, we propose the following.

Conjecture (Converse to Borel-Tits). Let $G$ be a reductive algebraic k-group which is anisotropic over $k$. Then every split spherical BN-pair for $G(k)$ is virtually trivial.

Recall that a BN-pair $(B, N)$ for a group $G$ is called spherical if the associated Weyl group $W:=N / T$ is finite, where $T:=B \cap N$. It is said to be split if it is saturated (i.e. $T=\bigcap_{w \in W} w B w^{-1}$ ), and if there exists a nilpotent normal subgroup $U \triangleleft B$ such that $B \cong U \rtimes T$. This implies that the associated building enjoys the Moufang property (see e.g. [11]). The BN-pair is called virtually trivial if the associated building is finite or, equivalently, if $B$ has finite index in $G$. The BN-pair $(P(k), N(k))$ for $G(k)$ described above is always split ([4, 14.19]). It is virtually trivial if and only if either $k$ is finite or $G$ is $k$-anisotropic. In particular, over infinite ground fields the conjecture can really be thought as a converse to the Borel-Tits theorem.

Besides the natural search for a converse to Borel-Tits, a motivation to consider the above conjecture is provided by the recent work of Peter Abramenko and Ken Brown [1], who constructed Weyl transitive actions on trees for certain anisotropic groups over global function fields. We refer to [2, Ch. 6] for more details on the relations and distinctions between BN-pairs, strong transitivity and Weyl transitivity.

Our first contribution concerns the special case when the ground field $k$ is a local field. The $k$-anisotropy of $G$ is then equivalent to the compactness of $G(k)$ (see [13]). In fact, our first step will be to establish the following two results, which concern arbitrary compact topological groups (not necessarily associated with algebraic groups). 
Theorem 1. Let $G$ be a compact group. Then every BN-pair for $G$ is spherical.

Theorem 2. Let $G$ be a compact group. Then every split spherical BN-pair for $G$ is virtually trivial.

We emphasize that the BN-pairs appearing in these statements are abstract: The corresponding subgroups $B$ and $N$ are not supposed to be closed in $G$. Specializing to anisotropic groups over local fields, we deduce the following immediate corollary.

Theorem 3. Let $k$ be a local field and $G$ be a connected semi-simple algebraic $k$-group which is anisotropic over $k$. Then:

(1) Every BN-pair for $G(k)$ is spherical.

(2) Every split spherical BN-pair $G(k)$ is virtually trivial.

Finally, we consider the case of perfect ground fields.

Theorem 4. Let $k$ be a perfect field and $G$ be a reductive algebraic $k$-group which is anisotropic over $k$. Then every split spherical BN-pair for $G(k)$ is virtually trivial.

Notice that Theorems 3 and 4 are logically independent, since there exist local fields which are not perfect and vice-versa.

It would be very interesting to sharpen the conclusion of Theorems 3 and 4, that is, to show that, under suitable assumptions, the BN-pair must be trivial, and not only virtually trivial. However, we expect this to be quite difficult, since it is closely related to a conjecture due to Andrei Rapinchuk and Gopal Prasad (see [14]), which may be stated as follows: "Let $G$ be a reductive $k$-group which is anisotropic over $k$. Then, every finite quotient of $G(k)$ is solvable." As of today, this conjecture was confirmed only when $G$ is the multiplicative group of a finite dimensional division algebra (see [15]). We now sketch informally how these two problems are related.

On one side, if $G(k)$ possesses a BN-pair with finite associated building $\Delta$, and if $K:=\operatorname{ker}(G(k) \curvearrowright \Delta)$ is the kernel of the corresponding action, then $G(k) / K$ is a finite group whose action on $\Delta$ is faithful, and thus $G(k) / K$ is a finite group which possesses a faithful BN-pair. But these groups have been classified: they are simple Chevalley groups, and in particular are not solvable (up to two exceptions). 
Thus, if the BN-pair for $G(k)$ were nontrivial, there would exist (modulo these two exceptions) a non-solvable finite quotient of $G(k)$.

Conversely, suppose that $G(k)$ possesses a nontrivial and non-solvable finite quotient $F^{\prime}:=G(k) / K$. Let $R \lessgtr F^{\prime}$ be the solvable radical of $F^{\prime}$, that is, its largest solvable normal subgroup. Going to the quotient $F:=F^{\prime} / R$, we thus know that $G(k)$ surjects onto a nontrivial finite group with trivial solvable radical (namely, $F$ ). Let now $M$ be a minimal normal subgroup of $F$. Then $M$ is a direct product of non-Abelian simple groups which are pairwise isomorphic, say $M \cong S_{1} \times \cdots \times S_{k}$ with $S_{i} \cong S$ for all $i \in\{1, \ldots, k\}$. By the classification of finite simple groups, $S$ is very likely to be a Chevalley group. Such a group possesses a root datum, and thus also a nontrivial BN-pair whose associated (finite) building is in bijection with $S / B$. Repeating this construction for each $S_{i}$, we then get a finite building $\Delta=\Delta_{1} \times \cdots \times \Delta_{k}$ on which $M=S_{1} \times \cdots \times S_{k}$ acts strongly transitively. Finally, the action of $\operatorname{Aut}(M)$ on the set of $p$-Sylow subgroups of $M$ (where $p=\operatorname{char} k$ ) induces an action of $\operatorname{Aut}(M)$ on $\Delta$ making the diagram

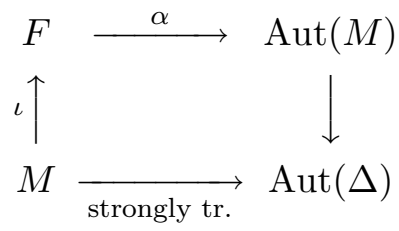

commute, where $\alpha(f)$ denotes the conjugation by $f$ for all $f \in F$. In particular, we get a strongly transitive action of $F$, and thus also of $G(k)$, on the finite building $\Delta$. This yields a nontrivial and virtually trivial BN-pair for $G(k)$.

General conventions. All algebraic groups considered here are supposed to be affine, all topological groups are assumed Hausdorff and all BN-pairs have finite rank.

\section{Proof of Theorem 1}

2.1. Heuristic sketch. Let $G$ be a compact group and let $(B, N)$ be a BN-pair for $G$. Let also $\Delta$ be the associated building. We consider the Davis realization of $\Delta$, noted $|\Delta|_{\mathrm{CAT}(0)}$ in this paper, and which is a complete CAT(0) space, as well as a simplicial complex, on which $G$ acts by simplicial isometries. The key step in the proof of Theorem 1 is to establish that this action is elliptic (Theorem 2.5 below). To do so, we use a result of Martin Bridson stating that such an action is always 
semi-simple, and we then argue by contradiction, assuming that $G$ possesses an element with no fixed point. Such an element would then generate a subgroup $Q$ of $G$ which acts by translations on $|\Delta|_{\mathrm{CAT}(0)}$. Moreover, the structure of simplicial complex of $|\Delta|_{\mathrm{CAT}(0)}$ implies that the set of translation lengths of the elements of $Q$ is discrete at 0 . The contradiction now comes from divisibility properties of compact and procyclic groups, which we apply to $Q$.

2.2. Procyclic groups. Let $G$ be a profinite group. Recall that $G$ is said to be procyclic if there exists a $g \in G$ such that the subgroup generated by $g$ is dense in $G$, that is, $G=\overline{\langle g\rangle}$. Moreover $G$ is said to be pro- $p$ for some prime $p$ if every finite Hausdorff quotient of $G$ is a $p$-group.

The following basic properties of procyclic groups can be found in [16, 2.7]. The symbol $\mathbb{P}$ denotes the set of all primes.

Proposition 2.1. Let $G$ be a procyclic group. Then,

(i) $G$ is the direct product $G=\prod_{p \in \mathbb{P}} G_{p}$ of its p-Sylow subgroups, and each $G_{p}$ is a pro-p procyclic group.

(ii) $G$ is, in a unique way, a quotient of $\hat{\mathbb{Z}}:=\prod_{p \in \mathbb{P}} \mathbb{Z}_{p}$. If $G$ is pro-p for some $p \in \mathbb{P}$, then it is a quotient of $\mathbb{Z}_{p}$.

2.3. Divisible groups. Recall that an element $g \in G$ is said to be $n$-divisible for some $n \in \mathbb{N}$ if there exists an $h \in G$ such that $h^{n}=g$. We say that $g$ is divisible if it is $n$-divisible for each $n \geq 1$. The group $G$ is called $n$-divisible (respectively divisible) when all its elements are.

Now, every prime $q$ different from $p$ is invertible in $\mathbb{Z}_{p}$ since its $p$-adic valuation is zero. Hence, the additive group $\mathbb{Z}_{p}$ is $q$-divisible for each $q \in \mathbb{P} \backslash\{p\}$. In particular, Proposition 2.1 implies that if a procyclic group $G$ has trivial $q$-Sylow subgroups, then $G$ is $q$-divisible.

We conclude this paragraph by stating the following characterization of divisibility for compact groups (see [12, Corollaire 2]).

Proposition 2.2. Let $G$ be a compact topological group. Then, $G$ is divisible if and only if it is connected.

2.4. Semi-simple actions on CAT(0) spaces. Let $G$ be a group acting on a metric space $(X, d)$. For every $g \in G$, we define the translation length of 
$g$ by $|g|:=\inf \{d(x, g \cdot x) \mid x \in X\} \in[0, \infty)$ and the minimal set of $g$ by $\operatorname{Min}(g):=\{x \in X|d(x, g \cdot x)=| g \mid\}$. An element $g \in G$ is said to be semisimple when $\operatorname{Min}(g)$ is nonempty. In that case, we say that $g$ is elliptic if it fixes some point, that is, if $|g|=0$; otherwise, if $|g|>0$, we call $g$ hyperbolic.

A geodesic line (respectively, geodesic segment) in $X$ is an isometry $f: \mathbb{R} \rightarrow$ $X$ (respectively, $f:[0 ; 1] \rightarrow X$ ); by abuse of language, we will identify $f$ with its image in $X$.

The following lemma follows from Proposition 2.4 in [6].

Lemma 2.3. Let $(X, d)$ be a complete $C A T(0)$ metric space, and let $C$ be a closed convex nonempty subset of $X$. Then:

(i) For every $x \in X$, there is a unique $y \in C$ such that $d(x, y)=d(x, C)$, where $d(x, C):=\inf _{z \in C} d(x, z)$. We call $y$ the projection of $x$ on $C$ and we write $y=\operatorname{proj}_{C} x$.

(ii) For all $x_{1}, x_{2} \in X$, we have $d\left(\operatorname{proj}_{C} x_{1}, \operatorname{proj}_{C} x_{2}\right) \leq d\left(x_{1}, x_{2}\right)$.

Suppose now that $(X, d)$ is a cell complex. We then say that $G$ acts by cellular isometries on $X$ if it preserves the metric, as well as the cell decomposition of $X$.

The following result is due to Martin Bridson [7].

Proposition 2.4. Let $X$ be a locally Euclidean CAT(0) cell complex with finitely many isometry types of cells, and $G$ be a group acting on $X$ by cellular isometries. Then every element of $G$ is semi-simple. Moreover, $\inf \{|g| \neq 0 \mid g \in G\}>0$.

We now establish the following result, which is the key ingredient for the proof of Theorem 1:

Theorem 2.5. Let $X$ be a locally Euclidean CAT(0) cell complex with finitely many isometry types of cells, and $G$ be a compact group acting on $X$ by cellular isometries (not necessarily continuously). Then every element of $G$ is elliptic.

Proof. Suppose for a contradiction there exists a $g \in G$ without fixed point. Proposition 2.4 then implies that $g$ is hyperbolic. Let $Q=\overline{\langle g\rangle}$ be the closure of the subgroup generated by $g$ in $G$. So, $Q$ is compact.

Claim 1: $Q$ is Abelian. 
This is clear since it contains a dense Abelian (in fact cyclic) subgroup.

Claim 2: For every $h \in Q$, the minimal set $\operatorname{Min}(h)$ is a closed convex subset of $X$ which is stabilized by $Q$.

This follows from [6, Proposition II.6.2].

Claim 3: For every $h \in Q$ and every nonempty closed convex subset $C$ of $X$ stabilized by $Q$, the set $C \cap \operatorname{Min}(h)$ is nonempty.

Note first that $\operatorname{Min}(h)$ is nonempty by Proposition 2.4. Let $x \in \operatorname{Min}(h)$ and consider the projections $y:=\operatorname{proj}_{C} x$ and $z:=\operatorname{proj}_{C} h x$ provided by Lemma 2.3. Since $h C=C$, we then obtain

$$
d(x, y)=\inf _{c \in C} d(x, c)=\inf _{c \in C} d(h x, h c)=\inf _{c \in C} d(h x, c)=d(h x, z) .
$$

Hence $d(h x, h y)=d(x, y)=d(h x, z)$, and so $z=h y=\operatorname{proj}_{C} h x$ by uniqueness of projections. Since in addition $d(y, z) \leq d(x, h x)=|h|$ by Lemma 2.3, we finally get $d(y, h y)=|h|$ and therefore $y \in C \cap \operatorname{Min}(h)$.

Claim 4: For all $h_{1}, h_{2} \in Q$, the set $\operatorname{Min}\left(h_{1}\right) \cap \operatorname{Min}\left(h_{2}\right)$ is nonempty.

As $\operatorname{Min}\left(h_{1}\right)$ and $\operatorname{Min}\left(h_{2}\right)$ are nonempty by Proposition 2.4, the claim follows from Claims 2 and 3.

Claim 5: Let $h \in Q$ and let $C$ be a nonempty closed convex subset of $X$ stabilized by $Q$. We may thus consider the action of $h$ on $C$. Denote by $|h|_{C}$ the translation length of $h$ for this action. Then, $h$ is semi-simple in $C$ and $|h|=|h|_{C}$.

Claim 3 yields that if $x \in \operatorname{Min}(h)$, then $y:=\operatorname{proj}_{C} x \in \operatorname{Min}(h)$. Since $\operatorname{Min}(h)$ is nonempty by Proposition 2.4, the claim follows.

Claim 6: For every $h \in Q$ and $n \geq 1$, we have $\left|h^{n}\right|=n|h|$.

By Claim 4, we may choose an $x \in \operatorname{Min}(h) \cap \operatorname{Min}\left(h^{n}\right)$. Note that $h$ is elliptic (respectively hyperbolic) if and only if $h^{n}$ is so (see [6, II.6.7 and II.6.8]). In particular, if $h$ is hyperbolic, then $x$ belongs to some $h$-axis, which is also an $h^{n}$-axis. In any case, we obtain $d\left(x, h^{n} x\right)=n d(x, h x)$, whence $\left|h^{n}\right|=d\left(x, h^{n} x\right)=$ $n d(x, h x)=n|h|$. 
Claim 7: Every divisible element of $Q$ is elliptic.

Let $h \in Q$ be divisible and suppose for a contradiction it is not elliptic. Then $h$ is hyperbolic by Proposition 2.4. For each natural number $n \geq 1$, choose an $h_{n} \in Q$ such that $h_{n}^{n}=h$. In particular, all $h_{n}$ are hyperbolic. Moreover, $\left|h_{n}^{n}\right|=n\left|h_{n}\right|$ by Claim 6. Therefore, we obtain a sequence $\left(h_{n}\right)$ of elements of $Q$ such that $\left|h_{n}\right|=|h| / n>0$, contradicting the second part of Proposition 2.4.

We now establish the desired contradiction to the hyperbolicity of $g$. First note that the component group $P:=Q / Q^{0}$ of $Q$ is a profinite group. In fact, it is even procyclic, since the subgroup generated by the projection of $g$ in $P$ is dense in $P$, the natural mapping $\pi: Q \rightarrow Q / Q^{0}$ being continuous. In particular, it follows from Proposition 2.1 that $P$ is the product of its $p$-Sylow subgroups $P_{p}$. Moreover, each $P_{p}$ is a pro- $p$ group and is therefore $q$-divisible for every $q \in \mathbb{P} \backslash\{p\}$. For each $p \in \mathbb{P}$, let $Q_{p}$ be the subgroup of $Q$ which is the pre-image of $P_{p}$ under $\pi$.

Claim 8: If $h, a, d \in Q$ with $h a=d^{n}$ for some $n \geq 1$ and $a$ is elliptic, then $|h|=n|d|$.

Write $C:=\operatorname{Min}(h) \cap \operatorname{Min}(a)$. Then $C$ is nonempty by Claim 4. Since $d^{n}$ stabilizes $C$, Claim 5 implies that it is semi-simple in $C$ with translation length $\left|d^{n}\right|_{C}=\left|d^{n}\right|$. Thus, $\left|d^{n}\right|_{C}=\left|d^{n}\right|=n|d|$ by Claim 6 . Note also that $h a$ is semi-simple in $C$ with translation length $|h a|_{C}=|h|$. Therefore, $|h|=|h a|_{C}=\left|d^{n}\right|_{C}=n|d|$, as desired.

Claim 9: Let $h \in Q$ be hyperbolic. Suppose that $h a_{i}=d_{i}^{n_{i}}$ for all $i \geq 1$, where $a_{i}, d_{i} \in Q$, each $a_{i}$ is elliptic and where $n_{i} \geq 1$. Then the set $\left\{n_{i} \mid i \geq 1\right\}$ is bounded.

Indeed, by Claim 8, the sequence $\left(d_{i}\right)$ of elements of $Q$ is such that $\left|d_{i}\right|=|h| / n_{i}>$ 0. The claim now follows from the second part of Proposition 2.4.

Claim 10: Let $p \in \mathbb{P}$. Then all elements of $Q_{p}$ are elliptic.

Suppose for a contradiction there exists an $h \in Q_{p}$ which is not elliptic, and is thus hyperbolic by Proposition 2.4. Let $q \in \mathbb{P} \backslash\{p\}$. Since $P_{p}=\pi\left(Q_{p}\right)$ is $q$-divisible, there exists an $h_{q} \in Q$ such that $h_{q}^{q} Q^{0}=h Q^{0}$. Let $a \in Q^{0}$ such that $h a=h_{q}^{q}$. By Proposition 2.2, since $Q^{0}$ is compact and connected, it is divisible, and so $a$ 
is elliptic by Claim 7. Since the set of natural prime numbers distinct from $p$ is unbounded, the desired contradiction now comes from Claim 9.

Let now $g Q^{0}=\left(g_{p}\right)_{p \in \mathbb{P}}$ be the decomposition of $\pi(g)$ in $P=\prod_{p \in \mathbb{P}} P_{p}$ (that is, each $\left.g_{p} \in P_{p}\right)$. Let $q \in \mathbb{P}$, and choose an $a_{q} \in Q_{p}$ such that $\pi\left(a_{q}\right)=g_{q}^{-1}$. Then $\pi\left(g a_{q}\right)$ has no component in the $q$-Sylow of $P$, and is therefore $q$-divisible in $P$. Hence, there exist an $h_{q} \in Q$ and an $a \in Q^{0}$ such that $g a_{q} a=h_{q}^{q}$. By Claim 10, we know that $a_{q}$ is elliptic. But so is $a$, and hence the product $a^{\prime}:=a_{q} a$ is also elliptic by Claim 4. Since $q$ is an arbitrary prime, Claim 9 again yields the desired contradiction.

2.5. The Davis realization of a building. We recall from [10] that any building $\Delta$ admits a metric realization, denoted by $|\Delta|_{\mathrm{CAT}(0)}$, which is a locally Euclidean CAT(0) cell complex with finitely many types of cells. Moreover any group of type-preserving automorphisms of $\Delta$ acts in a canonical way by cellular isometries on $|\Delta|_{\mathrm{CAT}(0)}$. Finally, the cell supporting any point of $|\Delta|_{\mathrm{CAT}(0)}$ determines a unique spherical residue of $\Delta$. In particular, an automorphism of $\Delta$ which fixes a point in $|\Delta|_{\mathrm{CAT}(0)}$ must stabilize the corresponding spherical residue in $\Delta$.

Here is a reformulation of Theorem 1.

Theorem 2.6. Let $G$ be a compact group acting strongly transitively by typepreserving automorphisms on a thick building $\Delta$. Then, $\Delta$ is spherical.

Proof. Let $(W, S)$ be the Coxeter system associated to $\Delta$, and let $\Sigma$ be the fundamental apartment of $\Delta$. Then, the action of the stabilizer in $G$ of $\Sigma$ can be identified with the action of $W$ on this apartment ([20, 2.8]).

Claim 1: $|\Sigma|_{\mathrm{CAT}(0)}$ is a closed convex subset of $|\Delta|_{\mathrm{CAT}(0)}$.

A basic fact about buildings is the existence, for each pair $(\Sigma, C)$ consisting of an apartment $\Sigma$ and of a chamber $C \in \Sigma$, of a retraction of $\Delta$ onto $\Sigma$ centered at $C$, that is, of a simplicial map $\rho=\rho_{\Sigma, C}: \Delta \rightarrow \Sigma$ preserving minimal galleries from $C$ and such that $\rho_{\mid \Sigma}=\operatorname{id}_{\mid \Sigma}$. The induced mapping $\bar{\rho}:|\Delta|_{\operatorname{CAT}(0)} \rightarrow|\Sigma|_{\operatorname{CAT}(0)}$ then maps every geodesic segment of $|\Delta|_{\mathrm{CAT}(0)}$ onto a piecewise geodesic segment of $|\Sigma|_{\operatorname{CAT}(0)}$ of same length. In particular, the mapping $\bar{\rho}$ is distance decreasing (see [10, Lemme 11.2]). Hence, if $x$ and $y$ are two points in $|\Sigma|_{\operatorname{CAT}(0)}$, then the geodesic segment from $x$ to $y$ is entirely contained in $|\Sigma|_{\operatorname{CAT}(0)}$ since its image by 
$\bar{\rho}$ is also a geodesic from $x$ to $y$. This proves that $|\Sigma|_{\operatorname{CAT}(0)}$ is convex. To see it is closed, it suffices to note that it is complete as a metric space since it is precisely the Davis realization of the building $\Sigma$.

Claim 2: If $g \in G$ is elliptic in $X=|\Delta|_{\mathrm{CAT}(0)}$ and stabilizes $|\Sigma|_{\mathrm{CAT}(0)}$, then $g$ is also elliptic in $|\Sigma|_{\mathrm{CAT}(0)}$.

This follows from Claim 5 in the proof of Theorem 2.5.

Theorem 2.5 now implies that the induced action of $W$ on $|\Sigma|_{\mathrm{CAT}(0)}$ is elliptic, that is, every $w \in W$ is elliptic. Notice that the $W$-action on $|\Sigma|_{\operatorname{CAT}(0)}$ is proper, since by construction, it is cellular and the stabilizer of every point is a spherical (in particular finite) parabolic subgroup of $W$. Recalling now that every infinite finitely generated Coxeter group contains elements of infinite order (in fact, so do all finitely generated infinite linear groups by a classical result of Schur [17]; in the special case of Coxeter groups, a direct argument may be found in [2, Proposition $2.74]$ ), we deduce that $W$ is finite. In other words $\Delta$ is spherical.

\section{Proof of Theorem 2}

3.1. Heuristic sketch. Let $G$ be a compact group possessing a split spherical BN-pair, and let $\Delta$ be the associated building. We first establish Theorem 2 when $G$ acts continuously on $\Delta$. In that case, 2-transitive actions (which are closely related to strongly transitive actions) of $G$ on subspaces $X$ of $\Delta$ are easily seen to be possible only for finite $X$. The second step is then to show that the action of $G$ on $\Delta$ has to be continuous. This uses the fact that buildings arising from split spherical BN-pairs are Moufang (see Proposition 3.3 below).

3.2. Continuous actions on buildings. Recall that a topological space $X$ is said to satisfy the $T_{1}$ separation axiom when all its singletons are closed. The following is probably well-known.

Lemma 3.1. Let $G$ be a compact group. If $G$ admits a continuous 2-transitive action on a $T_{1}$ topological space $X$, then $X$ is finite.

Proof. Define $Y:=\{(x, y) \in X \times X \mid x \neq y\} \subset X \times X$, and fix $x, y \in X$ with $x \neq y$. Since the orbit map $\alpha_{x}: G \rightarrow X: g \mapsto g \cdot x$ is continuous, so is 
$\alpha_{x} \times \alpha_{y}: G \rightarrow X \times X: g \mapsto(g \cdot x, g \cdot y)$. By 2-transitivity, we get $Y=\left(\alpha_{x} \times \alpha_{y}\right)(G)$, and so $Y$ is compact.

Note also that the mapping $f: X \times X \rightarrow X \times X:(a, b) \mapsto(x, b)$ is continuous. Setting $Z:=X \backslash\{x\}$, we then get $Z \times\{x\}=f^{-1}(\{(x, x)\}) \cap Y$, so that $Z \times\{x\}$ is closed in $Y$, and hence compact. It follows that $Z$ is compact, being the image of $Z \times\{x\}$ by the projection on the first factor $X \times X \rightarrow X$, which is of course continuous.

In particular, $Z$ is closed, and hence $\{x\}$ is open. It follows that $X$ is discrete, and therefore finite since $X=\alpha_{x}(G)$ is compact.

Let $\Delta$ be a building of type $(W, S)$, and denote by Ch $\Delta$ the set of its chambers. Consider the chamber system $\Gamma$ of $\Delta$, which is the labelled graph with vertex set Ch $\Delta$ and with an edge labelled by $s \in S$ for each pair of $s$-adjacent chambers of $\Delta$ (see [8, Ch.I Appendix D]). Let $J \subset S$. A $J$-gallery in $\Gamma$ between two chambers $x$ and $y$ of $\Delta$ is a sequence $\left(x=x_{0}, x_{1}, \ldots, x_{l}=y\right)$ of chambers of $\Delta$ such that for each $i \in\{1, \ldots, l\}$, there exists an $s \in J$ such that $x_{i-1}$ is $s$-adjacent to $x_{i}$. The natural number $l$ is called the length of the gallery. A minimal gallery is a gallery of minimal length. The distance in $\Delta$ between two chambers $x, y \in \mathrm{Ch} \Delta$ is the length of a minimal gallery joining $x$ to $y$. The diameter of $\Gamma$ is the supremum (in $\mathbb{N} \cup\{\infty\}$ ) of the distances between its vertices.

Let $J \subset S$. The $J$-residue $R=R_{J}(x)$ of some chamber $x \in \mathrm{Ch} \Delta$ is the set of chambers of $\Delta$ which are connected to $x$ by a $J$-gallery. When $J$ has cardinality 1 , we call $R$ a panel.

In this paper, we will say that a group $G$ acts continuously on $\Delta$ if the stabilizers of the residues of $\Delta$ are closed in $G$. Note that we can of course restrict our attention to the maximal proper residues, the others being obtained as intersections of those.

Lemma 3.2. Let $G$ be a compact group acting continuously and strongly transitively by type-preserving automorphisms on a spherical thick building $\Delta$. Then $\Delta$ is finite.

Proof. The stabilizer $H$ in $G$ of a panel $P$ of $\Delta$ is a closed and thus compact subgroup of $G$. 
Claim 1: $H$ acts 2-transitively on $\operatorname{Ch}(P)$.

Indeed, let $C$ be a chamber of $P$ and let $B:=\operatorname{Stab}_{G}(C) \subset H$. We first show that $B$, and thus also $H$, is transitive on the set $\mathcal{C}=P \backslash\{C\}$. Let $C_{1}, C_{2} \in \mathcal{C}$ and let $\Sigma_{1}$ (respectively, $\Sigma_{2}$ ) be an apartment containing $C$ and $C_{1}$ (respectively, $C$ and $C_{2}$ ). By strong transitivity, $B$ is transitive on the set of apartments containing $C$, and so there exists a $b \in B$ such that $b \Sigma_{1}=\Sigma_{2}$. Hence $b C_{1}=C_{2}$. It now remains to show that $H$ is transitive on $P$. But if $C_{1}, C_{2} \in P$, then since $\Delta$ is thick, we may choose a chamber $C$ in $P$ different from $C_{1}, C_{2}$. The stabilizer $B^{\prime}$ of $C$ in $G$ is then contained in $H$ and is transitive on $P \backslash\{C\}$ by the previous argument.

Now, identifying $\Delta$ with $\Delta(G, B)$, so that $H=B \cup B s B$ for some generator $s$ of the corresponding Weyl group, we get a 2-transitive, continuous action by left translation of the compact group $H$ on the topological space $H / B$. Moreover, this space is $T_{1}$ since $B$ is closed in $G$ by hypothesis. Lemma 3.1 then implies that $P$ is finite. In other words, as $P$ was arbitrary, the building $\Delta$ is locally finite, that is, every panel is finite. The following observation now allows us to conclude:

Claim 2: Every locally finite spherical building is finite.

Indeed, let $\Gamma=\mathrm{Ch} \Delta$ be the graph whose vertices are the chambers of $\Delta$, and such that two chambers of $\Delta$ are adjacent if they share a common panel. Since $\Delta$ is locally finite, so is $\Gamma$. Hence, fixing a vertex $x \in \Gamma$, each ball in $\Gamma$ centered at $x$ with radius $n(n \in \mathbb{N})$ possesses a finite number of vertices. Moreover, as $\Delta$ is spherical, the diameter of $\Delta$ is finite ([8, Ch.IV, 3]), and hence the diameter of $\Gamma$ is also finite. Thus $\Gamma$ is contained in such a ball, and is therefore finite.

3.3. Moufang buildings. Let $\Delta=\Delta(G, B)$ be the building associated to a split spherical BN-pair $(B=T \ltimes U, N)$ of type $(W, S)$. It is well-known (see the main result of [11]) that the existence of a splitting for the above BN-pair is equivalent to the fact that the building $\Delta$ enjoys the Moufang property, as defined in [20, Chapter 11].

Two chambers $x, y \in \mathrm{Ch} \Delta$ are called opposite if they are at maximal distance in the chamber system of $\Delta$. Similarly, one can define opposite residues (see for instance [2, 5.7]). The set of chambers (respectively, residues) of $\Delta$ which are opposite to a given chamber $C$ (respectively, residue $R$ ) will be denoted by $C^{\text {op }}$ (respectively, $R^{\mathrm{op}}$ ). 
Proposition 3.3. Let $P=B W_{J} B$ be a proper standard parabolic subgroup of $\Delta=\Delta(G, B)$ for some proper subset $J$ of $S$, let $C$ be the fundamental chamber (i.e. the unique chamber fixed by $B$ ) and let $R$ be the unique $J$-residue containing $C$. Define the subgroup $V:=\bigcap_{p \in P} p U p^{-1}$ of $G$. Then $V$ acts simply transitively on $R^{\mathrm{op}}$.

Proof. Let $\Sigma$ be an apartment containing $C$. By [20, 9.11], there exists a minimal galery $\gamma_{R^{\prime}}$, one for each residue $R^{\prime} \in R^{\mathrm{op}}$, beginning at $C$ and ending at a chamber $C^{\prime}$ in $R^{\prime}$ such that the type of $\gamma_{R^{\prime}}$ is independent of the choice of $R^{\prime}$ and $C=$ $\operatorname{proj}_{R} C^{\prime}$. Let $R^{\prime} \in R^{\text {op }}$ be the unique residue of $\Sigma$ opposite $R$ and let $C^{\prime}$ be the last chamber of $\gamma_{R^{\prime}}$. Let also $\alpha$ be a root of $\Sigma$ containing $C$ but not $C^{\prime}$. By [20, 8.21], $R \cap \Sigma \subset \alpha$. By [20, 9.7], therefore, $R$ is fixed pointwise by the root group $U_{\alpha}$. Since $P$ maps $R$ to itself, we have $C \in R \subset \alpha^{p}$ and hence $p^{-1} U_{\alpha} p \subset U$ for all $p \in P$ by the definition of root subgroups (see [20, 11.1]) and the fact that the 'radical' $U$ does not depend on the choice of the apartment $\Sigma$ (see [20, Proposition 11.11(iii)]). Thus $U_{\alpha} \subset V$. Now, as in [2, 7.67], one shows that the subgroup of $V$ generated by all $U_{\alpha}$ 's of the latter form acts transitively on the set $\left\{\gamma_{R^{\prime \prime}} \mid R^{\prime \prime} \in R^{\mathrm{op}}\right\}$, and hence also on $R^{\mathrm{op}}$.

Suppose $h \in V$ maps $R^{\prime} \in R^{\mathrm{op}}$ to itself. Then $h$ acts trivially on $R$. Since the restriction of $\operatorname{proj}_{R^{\prime}}$ to $R$ is a bijection from $R$ to $R^{\prime}$ (by [20,9.11] again), it follows that $h$ acts trivially on $R^{\prime}$. By [20,9.8], therefore, $h$ fixes two opposite chambers of $\Sigma$ and hence $h$ fixes $\Sigma$. By [20, 9.7] again, we conclude that $h=1$.

In particular, we have the following (compare $[8$, Ch.IV, 5]).

Lemma 3.4. Let $C$ be the fundamental chamber of $\Delta$. Then $U$ acts simply transitively on $C^{\mathrm{op}}$. Equivalently, $U$ acts simply transitively on the set of apartments containing $C$.

Lemma 3.5. Let $P=B W_{J} B$ be a proper standard parabolic subgroup of $\Delta=$ $\Delta(G, B)$ for some proper subset $J$ of $S$, let $C$ be the fundamental chamber and let $R$ be the unique $J$-residue containing $C$. Then there exist two chambers in $C^{\mathrm{op}}$ which are opposite to one another. In particular, $\left|R^{\mathrm{op}}\right| \geq 2$.

Proof. The first assertion holds by [2, Proposition 4.104] and the second follows since no proper residue contains two opposite chambers. 
We are now ready to complete the proof of Theorem 2 .

Theorem 3.6. Let $G$ be a compact topological group possessing a spherical split BN-pair $(B=T \ltimes U, N)$. Then the associated building is finite.

Proof. Let $\Delta=\Delta(G, B)$ be the building associated to $(B, N)$, and let $(W, S)$ be the corresponding Coxeter system.

We start with some basic observations in the case $(W, S)$ is not irreducible. Suppose thus that $S$ decomposes as $S=S_{1} \amalg S_{2}$ with $s_{1} s_{2}=s_{2} s_{1}$ for all $s_{1} \in S_{1}$ and $s_{2} \in S_{2}$. Then $W$ splits as a direct product $W \cong W_{1} \times W_{2}$, where $W_{i}=\left\langle S_{i}\right\rangle$, and the building $\Delta$ decomposes canonically as a product $\Delta=\Delta_{1} \times \Delta_{2}$ of buildings of type $\left(W_{1}, S_{1}\right)$ and $\left(W_{2}, S_{2}\right)$ respectively (see [20, Proposition 7.33]).

In particular, we obtain induced actions of $G$ on both $\Delta_{1}$ and $\Delta_{2}$, which are obviously strongly transitive. The corresponding BN-pairs for $G$ may be described as follows. Since each $s \in S$ can be written as a coset $n T \in N / T=W$, we may choose, for $i=1,2$, a set $\bar{N}_{i}$ of representatives in $N$ for the elements of $S_{i}$. For each $i=1,2$, consider now the subgroup $N_{i}$ of $N$ generated by $\bar{N}_{i}$ and $T$, and set $B_{i}:=\left\langle B \cup N_{3-i}\right\rangle=B N_{3-i} B \leq G$. Then $\left(B_{i}, N_{i}\right)$ is a spherical BN-pair for $G$, and the associated building is nothing but $\Delta_{i}=\Delta\left(G, B_{i}\right)$.

We claim that the BN-pair $\left(B_{i}, N_{i}\right)$ is split. This follows readily from the aforementioned equivalence between splittings of BN-pairs and the Moufang property for the associated buildings. More precisely, consider the group $U_{i}=\bigcap_{g \in B_{i}} g U g^{-1}$ which is the kernel of the $U$-action on $\Delta_{3-i}$. Then $U_{i}$ acts sharply transitively on the chambers of $\Delta_{i}$ which are opposite the standard chamber $C$, which by definition is the unique chamber fixed by $B_{i}$. Therefore we have $B_{i} \cong T_{i} \ltimes U_{i}$, where $T_{i}=\bigcap_{w \in W_{i}} w B_{i} w^{-1}$, and $U_{i}$ induces a splitting of the BN-pair $\left(B_{i}, N_{i}\right)$ as claimed.

This shows that the given split BN-pair for $G$ yields various split BN-pairs for $G$ corresponding to the various irreducible components of $\Delta$. Since $\mathrm{Ch} \Delta$ is naturally in one-to-one correspondence with the Cartesian product Ch $\Delta_{1} \times \cdots \times \mathrm{Ch} \Delta_{n}$ of the chamber sets of the various irreducible components of $\Delta$, the desired finiteness result readily follows provided we establish it for each irreducible BN-pair $\left(B_{i}, N_{i}\right)$ as above. In other words, there is no loss of generality in assuming that the building $\Delta$ is irreducible. We adopt henceforth this additional assumption. 
Let now $\mathcal{P}$ denote the set of maximal proper standard parabolic subgroups of $G$. Pick any $P \in \mathcal{P}$. Thus $P$ is of the form $P=B W_{J} B$ for some maximal subset $J \subsetneq S$, where $W_{J}=\langle J\rangle$. In particular, $P$ is a maximal subgroup of $G$ (see $[2$, Lemma 6.43(1)]). Define the normal subgroup

$$
V:=\bigcap_{p \in P} p U p^{-1} \unlhd P
$$

of $P$. As $V$ is contained in $U$, it is also nilpotent. Moreover, $V$ acts faithfully on $\Delta$. Indeed, the kernel $\operatorname{ker}(G \curvearrowright \Delta)$ of the action of $G$ on $\Delta$ is obviously contained in the stabilizer of the chambers of the fundamental apartment $\Sigma$, that is, in $\bigcap_{w \in W} w B w^{-1}=T$, and so

$$
V \cap \operatorname{ker}(G \curvearrowright \Delta) \subseteq U \cap T=\{1\} .
$$

Now, since $V$ is normal in $P$, we have $P \subseteq \mathscr{N}_{G}(V)$. Moreover, as the conjugation automorphism $\kappa_{g}: G \rightarrow G: x \mapsto g x g^{-1}$ is continuous, we get $\mathscr{N}_{G}(\bar{V}) \supseteq$ $\mathscr{N}_{G}(V)$ and so $\mathscr{N}_{G}(\bar{V}) \supseteq P$. Hence, by maximality of $P$, we obtain that either $\mathscr{N}_{G}(\bar{V})=P$ or $\mathscr{N}_{G}(\bar{V})=G$.

Claim: $\mathscr{N}_{G}(\bar{V})=P$ for all $P \in \mathcal{P}$.

Assume for a contradiction that $\mathscr{N}_{G}(\bar{V})=G$ for some $P \in \mathcal{P}$. In other words, $\bar{V} \triangleleft G$. In particular, the center $\mathscr{Z}(\bar{V}) \subseteq \bar{V}$ of $\bar{V}$ is also a normal subgroup of $G$. Moreover, $V$ is nontrivial since, by Proposition 3.3, it acts transitively on $R^{\mathrm{op}}$ and since $\left|R^{\mathrm{op}}\right| \geq 2$ by Lemma 3.5. As $V$ is nilpotent, this implies that $\mathscr{Z}(V)$ is also nontrivial.

Now, using again the continuity of the conjugation automorphism $\kappa_{h}$ (for $h \in$ $G)$, we see that $\mathscr{Z}(V)=\mathscr{Z}_{G}(V) \cap V$ is contained in $\mathscr{Z}(\bar{V})=\mathscr{Z}_{G}(\bar{V}) \cap \bar{V}$. Moreover, as $V$ acts faithfully on $\Delta$, so does $\mathscr{Z}(V)$. This implies in particular that $\mathscr{Z}(V)$, and thus also $\mathscr{Z}(\bar{V})$, act nontrivially on $\Delta$.

Tits' transitivity Lemma (see [8, Lemma 6.61]) then guarantees that the group $\mathscr{Z}(\bar{V})$ is transitive on the chambers of $\Delta$. In fact, this action is even simply transitive. Indeed, the stabilizers in $\mathscr{Z}(\bar{V})$ of the chambers of $\Delta$ are all conjugate by transitivity. They are thus all equal since $\mathscr{Z}(\bar{V})$ is Abelian, and are therefore contained in the kernel $\operatorname{ker}(G \curvearrowright \Delta)$ of the action of $G$ on $\Delta$. Since $\mathscr{Z}(V) \subseteq \mathscr{Z}(\bar{V})$, this implies that the action of $\mathscr{Z}(V)$ on Ch $\Delta$ is free. But since $\mathscr{Z}(V) \subseteq V \subseteq$ 
$U \subseteq B$, and as $B$ stabilizes the fundamental chamber, it follows that $\mathscr{Z}(V)$ acts trivially on $\Delta$. This contradiction establishes the Claim.

Since the normalizer of a closed subgroup is closed, we deduce from the Claim that every $P \in \mathcal{P}$ is closed. But this means that $G$ acts continuously on $\Delta$, and so Lemma 3.2 ensures that $\Delta$ is finite, as desired.

\section{Proof of Theorem 4}

Let $k$ be a perfect field and let $K=\bar{k}$ be its algebraic closure. In what follows, we identify an algebraic $k$-group $G$ with its group of $K$-rational points.

The main tool for the proof of Theorem 4 is the following result due to Borel and Tits (see [3]).

Proposition 4.1. Let $G$ be a reductive algebraic $k$-group and let $U$ be a unipotent $k$-subgroup of $G$. If $k$ is perfect, then there exists a parabolic $k$-subgroup $P$ of $G$ whose unipotent radical $R_{u}(P)$ contains $U$.

In particular, if $G$ is anisotropic over $k$, then $U$ must be trivial.

Proof of Theorem 4. Suppose for a contradiction that the split spherical BN-pair $(B, N)$ for the reductive $k$-group $G$ is such that $B$ has infinite index in $G(k)$. Let $\Delta=\Delta(G(k), B)$ be the associated building, and let $W$ be the corresponding (finite) Weyl group. Also, denote by $\bar{B}$ the Zariski closure of $B$ in $G$.

The Bruhat decomposition for $G$ yields $G=\coprod_{w \in W} B w B$. Since $G(k)$ is Zariski dense in $G$ by $[4,18.3]$, we have

$$
G=\overline{G(k)}=\overline{\coprod_{w \in W} B w B} \subseteq \coprod_{w \in W} \overline{B w B} .
$$

As $G$ is connected, it cannot be written as a finite union of closed subsets in a nontrivial way. Therefore, we deduce that $B w B$ is dense in $G$ for some $w \in W$. In particular, so is $\bar{B} w \bar{B}$.

Let now $A:=(\bar{B})^{0}$ be the identity component of $\bar{B}$. Since $A$ has finite index in $\bar{B}$, it follows that $\bar{B} w \bar{B}$ is a finite union of double cosets modulo $A$. As before, this implies that some double coset of the form $A z A$ is dense in $G$.

Claim: $\bar{B} \neq G$. 
Indeed, let $U$ be the nilpotent normal subgroup of $B$ arising from the splitting of the BN-pair, and suppose for a contradiction that $B$ is dense in $G$. Then the Zariski closure $\bar{U}$ of $U$ in $G$ is a nilpotent normal subgroup of $\bar{B}=G([4,2.1])$. Its identity component $\bar{U}^{0}$ is thus contained in the radical of $G$, which coincides with the connected center $\mathscr{Z}(G)^{0}([4,11.21])$. Hence, since $\bar{U}^{0}$ has finite index in $\bar{U}$, we get

$$
[U: U \cap \mathscr{Z}(G)] \leq\left[U: U \cap \bar{U}^{0}\right]=\left[U \bar{U}^{0}: \bar{U}^{0}\right] \leq\left[\bar{U}: \bar{U}^{0}\right]<\infty .
$$

Now, if $u \in U \cap \mathscr{Z}(G)$, then $u$ acts trivially on $\Delta$ since for any chamber $g B$, we have $u g B=g u B=g B$. As $U$ acts simply transitively on $C^{\text {op }}$ by Lemma 3.4, where $C=1_{G} B$ is the fundamental chamber of $\Delta$, this implies that $u=1$ : otherwise, $\Delta$ would contain only one apartment, so that $[G(k): B]<\infty$, a contradiction. So $U \cap \mathscr{Z}(G)=\{1\}$ and therefore $U$ is finite. Using again the sharp transitivity of $U$ on $C^{\text {op }}$, we deduce that $\Delta$ is the reunion of finitely many apartments, hence is finite, contradicting once more our initial hypothesis. The claim stands proven.

In particular $A$ is a proper closed connected subgroup of $G$ such that $A z A$ is dense in $G$ for some $z \in G$. The main result of [9] now implies that $A$ is not reductive, i.e. the unipotent radical $R_{u}(A)$ is nontrivial. Moreover, since $B$ is contained in $G(k)$ and is dense in $\bar{B}$, we know that $\bar{B}$ is defined on $k$ ([4, AG.14.4]). Hence, $A$ is also $k$-defined $([4,1.2])$, and so is $R_{u}(A)$ since $k$ is perfect ([18, 12.1.7(d)]). Thus $R_{u}(A)$ is a nontrivial unipotent $k$-subgroup of $G$. As remarked after Proposition 4.1 above, this contradicts the assumption that $G$ is anisotropic over $k$.

\section{REFERENCES}

[1] Peter Abramenko and Kenneth S. Brown. Transitivity properties for group actions on buildings. J. Group Theory, 10(3):267-277, 2007.

[2] Peter Abramenko and Kenneth S. Brown. Buildings, volume 248 of Graduate Texts in Mathematics. Springer, New York, 2008. Theory and applications.

[3] Armand Borel and Jacques Tits. Éléments unipotents et sous-groupes paraboliques de groupes réductifs. I. Invent. Math., 12:95-104, 1971.

[4] Armand Borel. Linear algebraic groups, volume 126 of Graduate Texts in Mathematics. Springer-Verlag, New York, second edition, 1991.

[5] Armand Borel and Jacques Tits. Groupes réductifs. Inst. Hautes Études Sci. Publ. Math., (27):55-150, 1965. 
[6] Martin R. Bridson and AndrÃĹ Haefliger. Metric spaces of non-positive curvature, volume 319 of Grundlehren der Mathematischen Wissenschaften. Springer-Verlag, Berlin, 1999.

[7] Martin R. Bridson. On the semisimplicity of polyhedral isometries. Proc. Amer. Math. Soc., 127(7):2143-2146, 1999.

[8] Kenneth S. Brown. Buildings. Springer Monographs in Mathematics. Springer-Verlag, New York, 1998. Reprint of the 1989 original.

[9] Jonathan Brundan. Double coset density in reductive algebraic groups. J. Algebra, 177(3):755-767, 1995.

[10] Michael W. Davis. Buildings are CAT(0). In Geometry and cohomology in group theory (Durham, 1994), volume 252 of London Math. Soc. Lecture Note Ser., pages 108-123. Cambridge Univ. Press, Cambridge, 1998.

[11] Tom De Medts, Fabienne Haot, Katrin Tent, and Hendrik Van Maldeghem. Split BN-pairs of rank at least 2 and the uniqueness of splittings. J. Group Theory, 8(1):1-10, 2005.

[12] Jan Mycielski. Some properties of connected compact groups. Colloq. Math., 5:162-166, 1958.

[13] Gopal Prasad. Elementary proof of a theorem of Bruhat-Tits-Rousseau and of a theorem of Tits. Bull. Soc. Math. France, 110(2):197-202, 1982.

[14] Andrei S. Rapinchuk. Algebraic and Abstract Simple Groups: Old ans New. Prepublication, pages 55-150, 2003.

[15] Andrei S. Rapinchuk, Yoav Segev, and Gary M. Seitz. Finite quotients of the multiplicative group of a finite dimensional division algebra are solvable. J. Amer. Math. Soc., 15(4):929978 (electronic), 2002.

[16] Luis Ribes and Pavel Zalesskii. Profinite groups, volume 40 of Ergebnisse der Mathematik und ihrer Grenzgebiete. 3. Folge. A Series of Modern Surveys in Mathematics. SpringerVerlag, Berlin, 2000.

[17] Issai Schur. Über Gruppen periodischer linearer Substitutionen. Berl. Ber., 1911:619-627, 1911.

[18] Tonny A. Springer. Linear algebraic groups, volume 9 of Progress in Mathematics. Birkhäuser Boston Inc., Boston, MA, second edition, 1998.

[19] Jacques Tits. Algebraic and abstract simple groups. Ann. of Math. (2), 80:313-329, 1964.

[20] Richard M. Weiss. The structure of spherical buildings. Princeton University Press, Princeton, NJ, 2003.

Pierre-Emmanuel Caprace

Université catholique de Louvain

Chemin du Cyclotron 2, 1348 Louvain-la-Neuve

Belgium

Email: pierre-emmanuel.caprace@uclouvain.be 
Timothée Marquis

Université libre de Bruxelles

Boulevard du Triomphe, 1050 Bruxelles

Belgium

Email: tmarquis@ulb.ac.be 